\title{
Investigation of droplet nucleation in CCS relevant systems: Progress in the design and testing of the mixture preparation device
}

\author{
Václav Vinš ${ }^{1, *}$, Miroslav Čenský1 ${ }^{1}$ Jan Hrubý ${ }^{1}$, and Jiř́ Hykl ${ }^{1}$ \\ ${ }^{1}$ Institute of Thermomechanics of the CAS, Dolejškova 1402/5, Prague 8, 182 00, Czech Republic
}

\begin{abstract}
The study presents progress in the development of mixture preparation device (MPD) representing an important part of the larger experimental setup intended for investigation of homogeneous droplet nucleation in $\mathrm{CO}_{2}$-rich systems. MPD allows for accurate adjustment of flow parameters, i.e. temperature, pressure, and flow rate, of $\mathrm{CO}_{2}$ in either superheated vapor or supercritical fluid phases and of other gas components such as argon or nitrogen. Through accurate settings of flow rates of individual components, the mixture composition can continuously be defined. MPD is going to be connected to the expansion chamber, where the droplet nucleation will experimentally be observed. In this work, $\mathrm{CO}_{2}$ branch, i.e. the core part of MPD, was modified and tested. Several components, e.g., pressure transducers and safety valve, had to be calibrated and adjusted to assure well-defined and safe operation. Most attention was paid to the design and performance of throttling capillary tubes installed in thermostatic bath, which define final flow parameters of $\mathrm{CO}_{2}$ coming from the $\mathrm{CO}_{2}$ branch. The flow characteristics of two capillary tubes with lengths of 7.8 and $4.0 \mathrm{~m}$ and inner diameter $0.1 \mathrm{~mm}$ were measured and compared to the predictions of a numerical model. The 1-D model of isothermal capillary flow was found to provide quite good agreement with the measured data.
\end{abstract}

\section{Introduction}

The present study is a continuation of a long-term research of the team from the Laboratory of Phase Transition Kinetics (LPTK) at the Institute of Thermomechanics of the CAS in Prague focused on investigation of droplet nucleation in $\mathrm{CO}_{2}$-rich systems $[1,2]$. The motivation is to generate new accurate data for the homogeneous nucleation of $\mathrm{CO}_{2}$ droplets in various systems relevant for CCS (carbon capture and storage) technologies [3], i.e. in $\mathrm{CO}_{2}$ mixtures with argon, nitrogen, and water. The new experimental data for the nucleation of $\mathrm{CO}_{2}$-rich mixtures shall help to validate theoretical models describing the phase transition processes relevant for $\mathrm{CO}_{2}$ transportation and storage.

The droplet nucleation is going to be investigated with the help of a rapid expansion, i.e. a sudden pressure drop, of the superheated vapor or supercritical fluid within an expansion chamber [4,5]. The apparatus is designed such that it allows for measurement of droplet nucleation over wide temperature range from $-20{ }^{\circ} \mathrm{C}$ to $+150{ }^{\circ} \mathrm{C}$ and pressures up to 130 bar. A unique experimental setup consists of three main parts: the mixture preparation device (MPD) intended for preparation of investigated mixtures [1], the expansion chamber with supporting expansion setup [2], and the optical stand for the investigation of droplet nucleation and growth using the light scattering.

This work focuses primarily on MPD, which is used for fine tuning of flow rates of individual components through the expansion chamber. An accurate adjustment of flow rates will be used for the definition of mixture composition. MPD consists of a set of flow controllers, pressure regulating valves, backpressure regulators, supercritical $\mathrm{CO}_{2}$ pump, and other equipment, which allow for fine adjustment of an overall mass flow rate of a given mixture from approximately 65 to $200 \mathrm{~g} /$ hour at pressures from 74 to 150 bar. Important elements of MPD are the throttling capillary tubes with inner diameter of around $0.1 \mathrm{~mm}$, which define and stabilize the $\mathrm{CO}_{2}$ flow under various conditions including the superheated vapor and the supercritical fluid. A numerical 1-D model similar to that developed in refs. [6 to 8] was employed for the prediction of flow rates through the capillary tubes under both the isothermal and adiabatic flow conditions. The individual components of MPD were further modified compared to previous study [1] and calibrated in order to improve the accuracy of the $\mathrm{CO}_{2}$ flow rate.

\section{Mixture preparation device}

The mixture preparation device is used for accurate definition of mixture composition flowing through the

\footnotetext{
Corresponding author: vins@it.cas.cz
} 
expansion chamber. The nucleation processes, i.e. onset of formation of a new phase (in our case droplets) from a metastable mother phase (mixture of carrying gas with superheated vapor or supercritical $\mathrm{CO}_{2}$ ), are in general extremely sensitive to a presence of impurities such as aerosol particles, mechanical impurities coming, e.g., from the tubing and fittings manufacture, or lubricant vapors. In order to minimize the effects of impurities and adsorption on inner walls of the apparatus, the gas mixture needs to flow through the expansion chamber for a longer period of time. Consequently, the mixture composition needs to be defined in a continuous way. Accurate tuning of flow rates of individual mixture components, e.g. of supercritical $\mathrm{CO}_{2}$ and gas nitrogen, allows for flushing possible impurities out of the apparatus and to equilibrate adsorption effects on the apparatus inner walls.

At the current state, MPD consists of two main parts: the $\mathrm{CO}_{2}$ branch intended for adjustment of pressure, temperature, and flow rate of either superheated vapor or supercritical fluid $\mathrm{CO}_{2}$ and the gas branch with nitrogen or argon.

\subsection{Nitrogen / argon gas branch}

In our experiments, nitrogen or eventually argon are considered as carrying gases, i.e. admixture components, which play a crucial role during abrupt pressure drop connected with the temperature decrease inside the expansion chamber. However, the carrying gas itself does not change its phase during the expansion and has only additional influence on the nucleation of droplets of the main component, i.e. $\mathrm{CO}_{2}$ in our case.

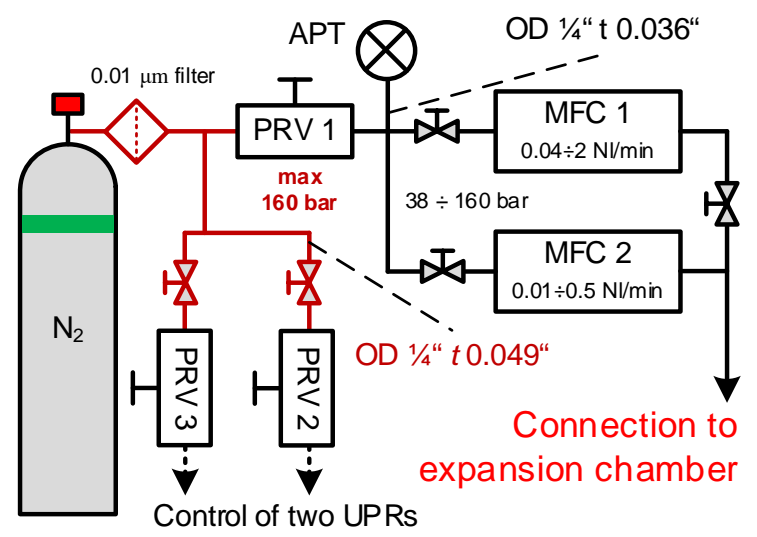

Fig. 1. Scheme of the gas branch of MPD: PRV - pressure regulating valve, APT - absolute pressure transducer, MFC - mass flow controller, UPR - upstream pressure regulator.

A simplified scheme of the gas branch is depicted in Fig. 1. The carrying gas is supplied from a gas cylinder with filling pressure either 200 or 300 bar, which is set to a desired value with the pressure regulating valve (PRV 1). The output pressure varies between 38 and 160 bar. The flow rate of either nitrogen or argon is defined with the help of two mass flow controllers MFC 1 and MFC 2 with different ranges of up to 2 $\mathrm{Nl} / \mathrm{min}$ and $0.5 \mathrm{Nl} / \mathrm{min}$, respectively. A relatively large range of gas flow rates can be adjusted using MFCs either individually or in parallel. Additionally, the gas bottle is connected to two other pressure reduction valves, which are used for the set point adjustment on two upstream pressure regulators (UPR) employed in other part of MPD in the expansion chamber section.

\section{$2.2 \mathrm{CO}_{2}$ branch}

$\mathrm{CO}_{2}$ branch represents the core part of MPD as it defines flow conditions of $\mathrm{CO}_{2}$ in either superheated vapor phase or supercritical fluid phase. The preliminary design and functionality of $\mathrm{CO}_{2}$ branch were described in previous study [1]. In this work, the setup was further modified, tested, and calibrated.

A connection scheme of the current design is shown in Fig. 2. $\mathrm{CO}_{2}$ in the form of saturated liquid at a pressure of around 63 bar corresponding to the ambient temperature $24{ }^{\circ} \mathrm{C}$ is supplied from $\mathrm{CO}_{2}$ cylinder with a dip tube (1). The saturated liquid is pressurized and pumped with the supercritical fluid pump (SCP, item 3) to pressures up to 310 bar, which is the safety limit of the setup. SCP can deliver flow rate of saturated liquid $\mathrm{CO}_{2}$ from 0.01 to $24 \mathrm{ml} / \mathrm{min}$ at pressures up to $690 \mathrm{bar}$. In order to stabilize possible pressure and flow rate fluctuations, the pressurized $\mathrm{CO}_{2}$ flows in a buffer volume realized as an accumulator AC (4) with inner volume of $150 \mathrm{ml}$. The accumulator is equipped with a safety valve (5) set to an opening pressure of 307 bar. Setting of the safety valve is discussed in section 3.1. in more detail. From the accumulator, the supercritical $\mathrm{CO}_{2}$ can flow either through throttling adiabatic capillary tube CAP 2 back to the low-pressure line before SCP or further into the MPD setup. Capillary tube CAP 2 assures continuous flow regime of SCP even when other parts of the $\mathrm{CO}_{2}$ branch or eventually of entire MPD are closed or under transient flow conditions. Pressure in the high-pressure line is measured with pressure transducer (6). Temperature and pressure of $\mathrm{CO}_{2}$ can be varied with the help of two stainless steel capillary tubes CAP 3 and CAP 4 submerged in a thermostatic bath (7) with liquid water. Both capillary tubes have inner diameter of around $0.10 \mathrm{~mm}$ and lengths of $7.8 \mathrm{~m}$ and $4.0 \mathrm{~m}$ in case of CAP 3 and CAP 4, respectively. Pressure drop on both capillary tubes is measured with differential pressure transducer (9) and the outlet pressure with absolute pressure transducer (8). After the absolute pressure transducer (8), the $\mathrm{CO}_{2}$ branch is going to be connected to the other parts of MPD in the final design. However, in order to test and calibrate the $\mathrm{CO}_{2}$ branch the current setup is equipped with a set of additional components at the low-pressure line. The mass flow of $\mathrm{CO}_{2}$ is currently measured with Coriolis mass flow meter (10) with range up to $200 \mathrm{~g} / \mathrm{h}$. The output pressure of isothermal capillary tubes CAP 3 and CAP 4 can be adjusted with an upstream pressure regulator (UPR, item 11), after which $\mathrm{CO}_{2}$ reaches the saturated liquid conditions and flows back to the inlet line from the liquid cylinder (1). Set point of UPR is defined with pressure of nitrogen gas (13) adjusted with the pressure regulating valve (PRV) with manometer (12). 


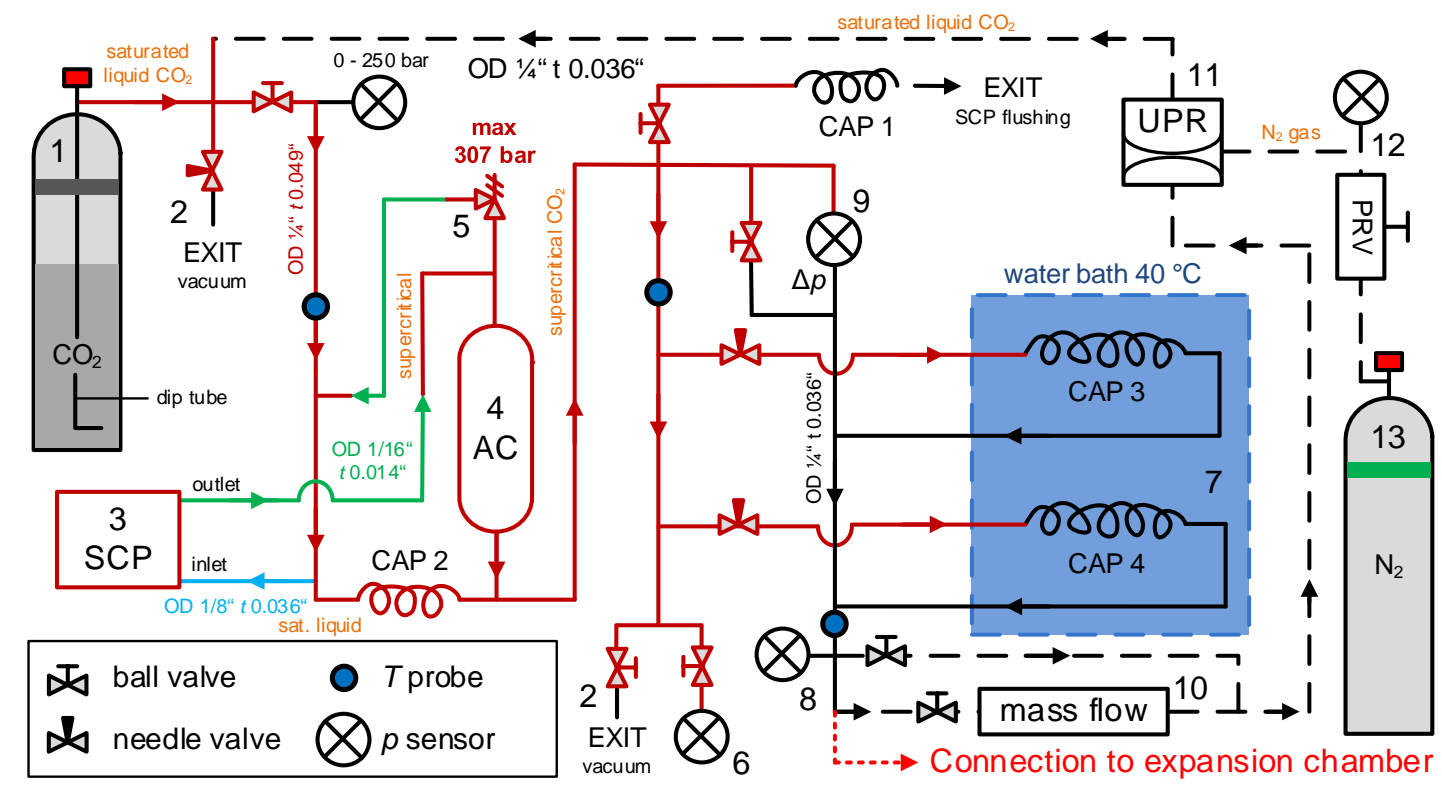

Fig. 2. Scheme of $\mathrm{CO}_{2}$ branch on MPD setup: 1 - liquid $\mathrm{CO}_{2}$ cylinder, 2 - connections to vacuum pump, 3 - supercritical fluid pump (SCP), 4 - accumulator (AC), 5 - safety valve, 6 - absolute pressure transducer on high pressure side, 7 - water thermostatic bath, 8 -absolute pressure transducer on low pressure side, 9 -differential pressure transducer measuring pressure drop over isothermal capillary tubes CAP 3 and CAP 4 in liquid bath, 10 - Coriolis mass flow meter, 11 - upstream pressure regulator (UPR), 12 - pressure regulating valve (PRV) with manometer, 13 - gas $\mathrm{N}_{2}$ cylinder.

Employment of accurate UPR represents one of the main modifications compared to the original design, where a throttling capillary tube either open to ambient or connected back to the inlet saturated liquid line was used; see Fig. 1 in ref. [1]. Temperature of $\mathrm{CO}_{2}$ is measured with a set of Pt100 temperature probes attached on the tubbing surface under the thermal insulation foam. It is assumed that the temperature of the insulated tubing is close to the temperature of flowing $\mathrm{CO}_{2}$.

In case the $\mathrm{CO}_{2}$ branch is open to ambient air, the setup has to be vacuumed using two vacuum connections (2) before the $\mathrm{CO}_{2}$ refilling. Approximately 20 minutes flushing with saturated liquid $\mathrm{CO}_{2}$ is required before the SCP start in this case. The exhaust capillary tube CAP 1 has to be open during the flushing.

\section{Improvements of $\mathrm{CO}_{2}$ branch}

The $\mathrm{CO}_{2}$ branch of the MPD setup was modified compared to its preliminary design described in previous study [1]. Main improvement necessary for the calibration of capillary tubes represents installation of UPR on the low-pressure line, which allows for accurate definition of the outlet pressure after the thermostatic bath (7) in a wide range of pressures from approximately 67 to 150 bar. Besides, the safety valve on the accumulator (4), the pressure transducers, and other components were carefully adjusted and calibrated.

\subsection{Calibration of differential $p$-transducer}

In the final application of the $\mathrm{CO}_{2}$ branch, the outlet pressure and temperature will primarily be set with the help of capillary tubes CAP 3 and 4 placed in the thermostatic bath (item 7 in Fig. 2). The pressure drop on the capillary tubes will be measured with the differential pressure transducer (9). Consequently, the pressure transducer had to be carefully calibrated. Fig. 3 shows the calibration curve for the differential pressure transducer in the pressure range $\Delta p=27 \div 185$ bar. A linear calibration curve with almost negligible scattering was obtained for the differential pressure transducer (9). Settings on the electrical demodulator of the pressure transducer were 5.12 for "zero" and 3.985 for "span". We note, that absolute pressure transducers 6 and 8 are planned to be removed from the $\mathrm{CO}_{2}$ branch and placed in other sections of MPD in the final design.

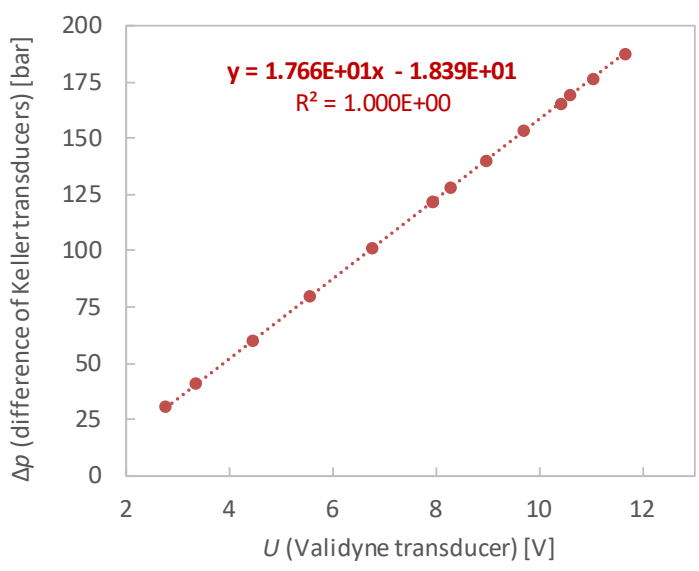

Fig. 3. Calibration curve for differential pressure transducer (DPT) measuring pressure drop over isothermal capillary tubes within liquid bath. 


\subsection{Settings of safety valve}

As already mentioned, the $\mathrm{CO}_{2}$ branch shall operate at a maximum pressure of around 310 bar. Main limitation is the maximum allowed pressure of the accumulator (4), which is 344 bar at the considered temperatures close to ambient. Even though a maximum allowed pressure can be set on the SCP pump with a constant flow rate, an additional safety precaution has to be applied on the $\mathrm{CO}_{2}$ branch. Therefore, a safety valve (5) was connected to the accumulator. The valve allows for adjustment of opening pressure, called also the cracking pressure, in the range from 276 bar to 345 bar by adjusting compression of a spring within the valve.

Fig. 4 shows results for the settings of the safety valve. The maximum pressure within the $\mathrm{CO}_{2}$ branch marked as ' $p$ after AC' increases with increasing flow rate pre-set on SCP. At lower pressures, the safety valve is closed and $\mathrm{CO}_{2}$ from the accumulator (4) flows partly through the backflow capillary tube CAP 2 and partly through isothermal capillary tube CAP 4 . CAP 3 was closed during the tests. The mass flow measured by flow meter (10), denoted as ' $m$ CORIflow' increases accordingly in this case.

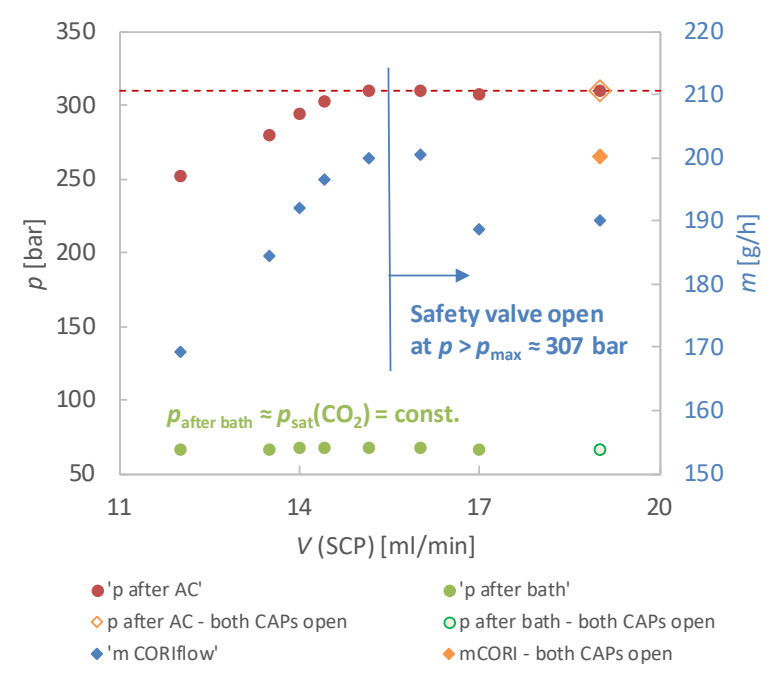

Fig. 4. Settings of safety valve installed on accumulator (AC) for a maximum pressure of 307 bar.

At pressure around $307 \mathrm{bar}$, the safety valve reaches its cracking pressure and gradually opens. Under these conditions, the supercritical $\mathrm{CO}_{2}$ from the accumulator flows not only through the backflow capillary tube CAP 2 and the isothermal capillary tube CAP 4 but part of it goes back to the SCP low pressure inlet line through the safety valve. As $\mathrm{CO}_{2}$ delivered by SCP flows newly through three lines instead of two, the measured mass flow through isothermal capillary tube CAP 4 slightly drops as can be seen in Fig. 4 at the highest SCP volumetric flow rates of 17 and $19 \mathrm{ml} / \mathrm{min}$. We note, that pressure after the Coriolis mass flow meter (10) denoted as ' $p$ after bath' was constant equal approximately to the saturated pressure of $\mathrm{CO}_{2}$ at ambient temperature around $24{ }^{\circ} \mathrm{C}$ during all measurements.
An additional case with both isothermal capillary tubes CAP 3 and CAP 4 open is also shown in Fig. 4 for the highest SCP flow rate of $19 \mathrm{ml} / \mathrm{min}$. As can be seen, the mass flow through both capillary tubes increased only slightly to $200 \mathrm{~g} / \mathrm{h}$ compared to $190 \mathrm{~g} / \mathrm{h}$ with only CAP 4 open. This shows that the flow rate pre-set on SCP was not sufficient to fully feed all three lines in this case. As will be shown in section 3.3, at the high pressure of 300 bar and low pressure around 67 bar, CAP 3 with a length of $7.8 \mathrm{~m}$ shall deliver roughly $80 \mathrm{~g} / \mathrm{h} \mathrm{CO} \mathrm{CO}_{2}$ and CAP 4 with a length of $4.0 \mathrm{~m}$ approximately $190 \mathrm{~g} / \mathrm{h}$. Consequently, both capillary tubes shall deliver around $270 \mathrm{~g} / \mathrm{h} \mathrm{CO}_{2}$ under these conditions and not only $200 \mathrm{~g} / \mathrm{h}$.

\subsection{Flow regimes in isothermal capillary tubes}

Appropriate design and correct understanding of flow regimes within a set of capillary tubes employed along the $\mathrm{CO}_{2}$ branch appear to be crucial for proper functionality of the entire system. Consequently, a considerable part of our experimental investigation was dedicated to the capillary tubes.

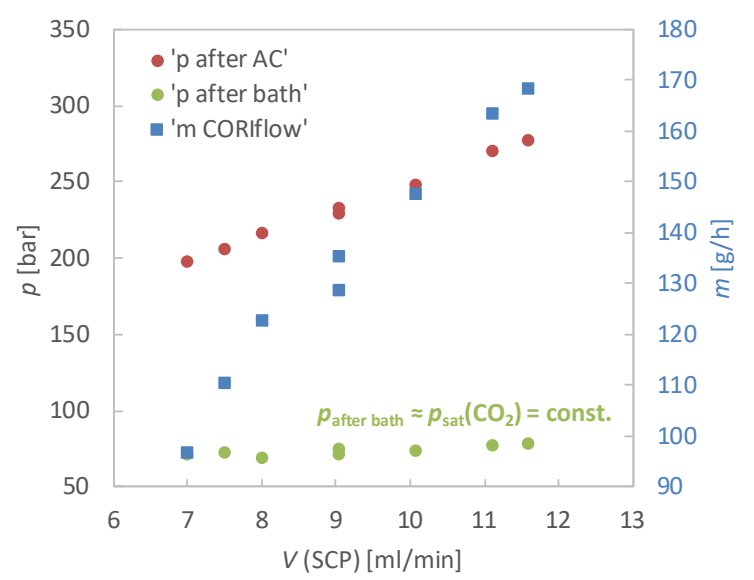

Fig. 5. Inlet pressure ( $p$ after AC), outlet pressure ( $p$ after bath) and mass flow rate through capillary tube CAP 3 ( $d=0.01 \mathrm{~mm}, L=7.8 \mathrm{~m}$ ) depending on flow rate set on the SCP pump.

Performance of capillary tubes CAP 1 and CAP 2 intended for system flushing and as backflow tube for stable flow conditions of SCP, respectively, was partly discussed in the previous work [1]. The output flow rate from $\mathrm{CO}_{2}$ branch is defined by isothermal capillary tubes CAP 3 and CAP 4 placed in the thermostatic water bath (7). Fig. 5 and Fig. 6 show flow characteristics of these capillary tube. The inlet and outlet pressures together with the mass flow rate through the capillary tubes are plotted depending on the SCP set point. With increasing flow rate set on SCP, the inlet pressure and the mass flow increase almost linearly. The outlet pressure was kept constant at a value close to the saturated pressure at the ambient temperature.

From the comparison of flow characteristics in Fig. 5 and Fig. 6, one can see an interesting but unwelcome experimental feature. Capillary tube CAP 3 with a length 
of $7.8 \mathrm{~m}$ delivers considerably high flow rate of around $140 \mathrm{~g} / \mathrm{h}$ compared to capillary tube CAP 4 with an almost half length of $4.0 \mathrm{~m}$, which delivers approximately $170 \mathrm{~g} / \mathrm{h}$. This discrepancy points on some sort of an experimental failure. We suspect that the needle valve before capillary tube CAP 4 was not entirely closed during the tests of capillary tube CAP 3. This assumption is supported by other experimental tests shown in Fig. 7, when the output pressure was varied with a help of the upstream pressure regulator (UPR, item 11 in Fig. 2). The mass flow rate through capillary tube CAP 3 varied around $75 \mathrm{~g} / \mathrm{h}$ in this case. Consequently, the mass flow through CAP 3 around $140 \mathrm{~g} / \mathrm{h}$ shown in Fig. 5 is incorrect.

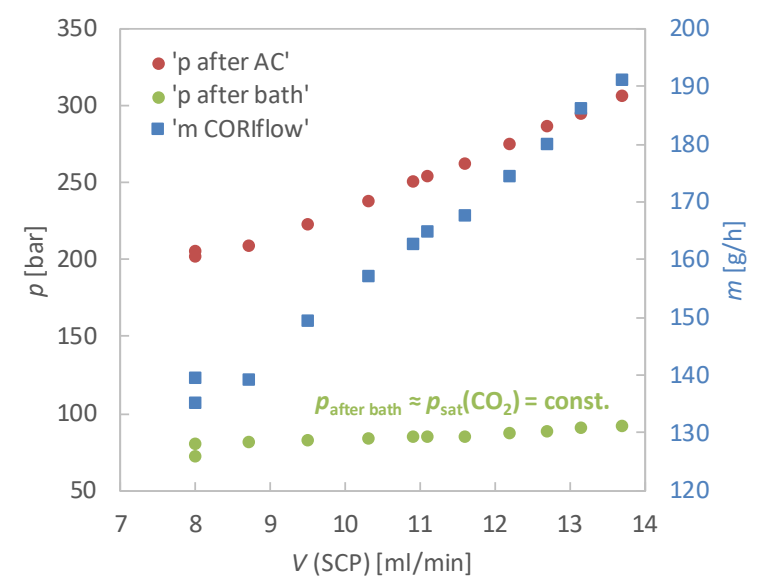

Fig. 6. Inlet pressure ( $p$ after AC), outlet pressure ( $p$ after bath) and mass flow rate through capillary tube CAP 4 ( $d=0.01 \mathrm{~mm}, L=4.0 \mathrm{~m}$ ) depending on flow rate set on the SCP pump.

Fig. 7 shows pressure drop on capillary tube CAP 3 and the outlet pressure varied by UPR depending on the mass flow. The experimental data are compared to the predictions of a 1-D numerical model (empty symbols). The model of an isothermal capillary flow is similar to the numerical models developed in refs. [6 to 8]. Properties of $\mathrm{CO}_{2}$ were calculated using the REFPROP package [9]. The equation of state by Span and Wagner [10] was used to calculate density depending on temperature and pressure. Viscosity of $\mathrm{CO}_{2}$ was calculated from the equation by Fenghour et al. [11]. The average inner diameter of capillary tube CAP 3 of $0.095 \mathrm{~mm}$ was considered to be slightly lower than the value given by the manufacturer of $0.10 \mathrm{~mm}$. The capillary tubes are manufactured with the drawing process and by such small tubes a variation of inner diameters better than $\pm 20 \%$ can only hardly be avoided. A relative inner wall roughness of 0.005 was employed in the model.

As can be seen in Fig. 7, the numerical model is in fairly good agreement with the experimental measurements. The mass flow through capillary tube CAP 3 lies around $75 \mathrm{~g} / \mathrm{h}$, which is far below the flow rate given in Fig. 5. The agreement of new experiment shown in Fig. 7 and the numerical model verifies potential opening of the needle valve by capillary tube CAP 4 as discussed earlier by Fig. 5.

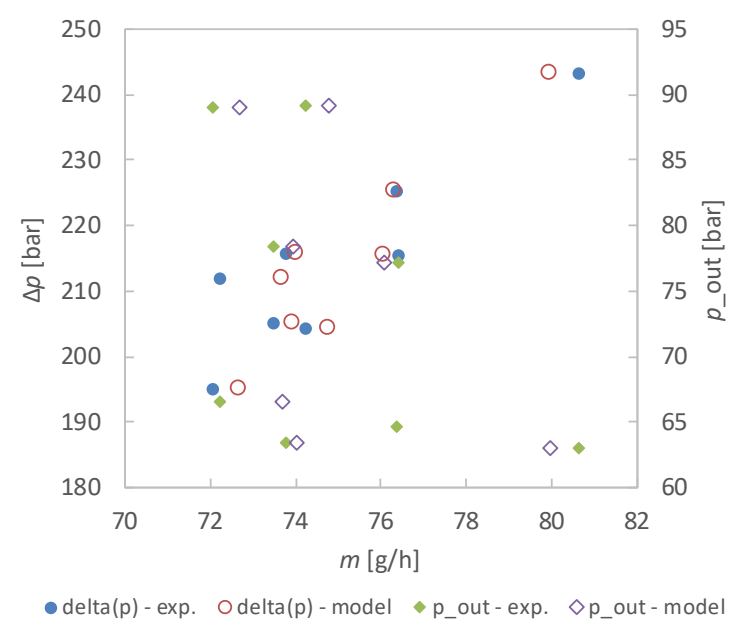

Fig. 7. $\mathrm{CO}_{2}$ mass flow rate through isothermal capillary tube CAP $3(d=0.095 \mathrm{~mm}, L=7.8 \mathrm{~m})$ versus pressure drop over the capillary tube and outlet pressure after the capillary tube. Experimental data versus model.

Fig. 8 shows results for shorter capillary tube CAP 4. The model predictions are in very good agreement with the measurements in this case over entire mass flow rate range from $168 \mathrm{~g} / \mathrm{h}$ to $200 \mathrm{~g} / \mathrm{h}$. The inner diameter of capillary tube was estimated to be of around $0.1165 \mathrm{~mm}$. We note that the capillary tube inner diameter is not constant along its length. Moreover, additional effects such as coiling or squeezing of the tube ends can also influence overall performance of the capillary tube. On the other hand, in the numerical model a constant value for the inner diameter is considered.

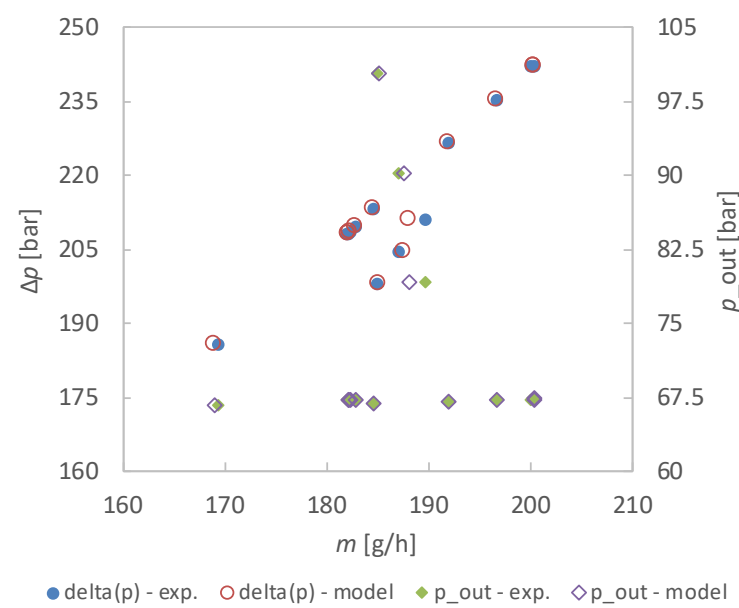

Fig. 8. $\mathrm{CO}_{2}$ mass flow rate through isothermal capillary tube CAP $4(d=0.1165 \mathrm{~mm}, L=4.0 \mathrm{~m})$ versus pressure drop over the capillary tube and outlet pressure after the capillary tube. Experimental data versus model.

Main output of results provided in Fig. 7 and Fig. 8 is that the numerical model can successfully be employed on the prediction of $\mathrm{CO}_{2}$ flow within isothermal capillary tubes. In further step, experimental tests with capillary 
tube CAP 3 shown in Fig. 5 will be repeated in order to clarify potential failure of the open needle valve by capillary tube CAP 4. Next, the performance of the backflow capillary tube CAP 2 will also be investigated and compared to the predictions of the adiabatic numerical model.

\section{Conclusions}

The modifications and improvements of the mixture preparation device (MPD) has been described in this work. Especially, the $\mathrm{CO}_{2}$ branch representing the core part of MPD was considerably improved. A calibration curve for the differential pressure transducer was obtained. The safety valve assuring maximum operation pressure of 307 bar was carefully set and tested. Most attention was dedicated to performance of isothermal capillary tubes defining the final flow parameters of $\mathrm{CO}_{2}$ entering other parts of MPD and subsequently the expansion chamber. Experimental results for the capillary flow were compared to predictions of a 1-D numerical model. Quite good agreement was achieved between the predicted mass flow and the experimental tests. Consequently, the model can be used to predict various operating conditions of the capillary tubes.

In further steps, the performance of the backflow capillary tube CAP 2 will be investigated and the $\mathrm{CO}_{2}$ branch is going to be linked with the gas branch operating with nitrogen or argon. These two branches represent initial design of MPD that can be connected to the setup with expansion chamber.

\section{Acknowledgement}

The study was supported by the Ministry of Education, Youth and Sports of the Czech Republic under OP RDE grant number CZ.02.1.01/0.0/0.0/16 019/0000753 "Research centre for lowcarbon energy technologies" and by the institutional support RVO:61388998 from IT CAS.

\section{References}

1. V. Vinš, M. Čenský, J. Hykl, J. Hrubý, EPJ Web Conf. 143, 02140 (2017)

2. M. Čenský, J. Hrubý, V. Vinš, J. Hykl, B. Šmíd, EPJ Web Conf. 180, 02015 (2018)

3. IPCC Special Report: Carbon Dioxide Capture and Storage - Summary for Policymakers. Intergovernmental Panel on Climate Change, Eighth Session of IPCC Working Group III, Montreal, (Canada) 22-24 September 2005

4. J. Wölk, R. Strey, J. Phys. Chem. B 105, 1168311701 (2001)

5. B.M. Cwilong, M.H. Edwards, Phys. Rev. 85, 380381 (1952)

6. V. Vacek, V. Vinš, Int. J. Thermophys. 30, 11791196 (2009)

7. V. Vacek, V. Vinš, Int. J. Thermophys. 28, 14901508 (2007)

8. V. Vinš: Mathematical modeling and experimental investigation of the two-phase flow through small- diameter tubes, Ph.D. dissertation, Czech Technical University in Prague, Czech Republic, 2009, doi: 10.13140/RG.2.2.31422.48967

9. E.W. Lemmon, M.L. Huber, M.O. McLinden, REFPROP, NIST Standard Reference Database 23 Version 9.1 (2013)

10. R. Span, W. Wagner, J. Phys. Chem. Ref. Data 25, 1509-1596 (1996)

11. A. Fenghour, W.A. Wakeham, V. Vesovic, J. Chem. Ref. Data 27, 31-44 (1998) 